\title{
Fluorescent carbon and graphene oxide nanoparticles synthesized by the laser ablation in liquid
}

\author{
A. Małolepszy ${ }^{1} \cdot$ S. Błonski ${ }^{2} \cdot$ J. Chrzanowska-Giżyńska ${ }^{2} \cdot$ M. Wojasiński ${ }^{1} \cdot$ T. Płocinski $^{3} \cdot$ L. Stobinski $^{1} \cdot$ Z. Szymanski $^{2}$
}

Received: 20 October 2017 / Accepted: 2 March 2018 / Published online: 8 March 2018

(c) The Author(s) 2018. This article is an open access publication

\begin{abstract}
The results of synthesis of the fluorescent carbon dots (CDots) from graphite target and reduced graphene oxide (rGO) nanoparticles performed by the nanosecond laser ablation in polyethylene glycol $200\left(\mathrm{PEG}_{200}\right)$ are shown. Two-step laser irradiation (first graphite target, next achieved suspension) revealed a very effective production of CDots. However, the ablation in PEG appeared to be effective with $1064 \mathrm{~nm}$ laser pulse in contrast to the ablation with $355 \mathrm{~nm}$ laser pulse. In the case of rGO nanoparticles similar laser irradiation procedure was less efficient. In both cases, received nanoparticles exhibited strong, broadband photoluminescence with a maximum dependent on the excitation wavelength. The size distribution for obtained CDots was evaluated using the DLS technique and HRTEM images. The results from both methods show quite good agreement in nanoparticle size estimation although the DLS method slightly overestimates nanoparticle's diameter.
\end{abstract}

\section{Introduction}

Pulsed laser ablation in liquid (PLAL) is a widely recognized method of synthesizing contamination-free nanostructures especially nanoparticles $[1,2]$ among them fluorescent carbon nanoparticles (FCNPs) [3], carbon polyynes [4], etc. Most of the attention is given to fluorescent nanoparticles, especially with diameters of $2-8 \mathrm{~nm}$ called carbon dots, which are widely used for purposes of fluorescent imaging, mainly of cells and tissues [5, 6]. Their most remarkable property is the excitation-dependent fluorescence emission. The laser synthesis of fluorescent carbon nanoparticles was performed in several liquids such as deionized water [7], isopropyl alcohol (IPA), acetone [8], polyethylene glycol (PEG) [5], ethylenediamine (EDA) and ionic liquids [9]. In the case of water, the fluorescent carbon dots are obtained

\section{Z. Szymanski}

zszym@ippt.pan.pl

1 Faculty of Chemical and Process Engineering, Warsaw University of Technology, Warynskiego 1, 00-645 Warsaw, Poland

2 Institute of Fundamental Technological Research, Polish Academy of Sciences, Pawinskiego 5B, 02-106 Warsaw, Poland

3 Faculty of Materials Science and Engineering, Warsaw University of Technology, Wołoska 141, 02-507 Warsaw, Poland only after further functionalization, either with the use of UV radiation [7] or by attaching simple organic species to the acid-treated carbon particles [5].

A comprehensive review of laser synthesis of nanoparticles in liquids has previously been presented [2].

Nanoparticles (NPs) approved in preclinical studies for both imaging and therapy often include polyethylene glycol. Attachment of PEG (PEGylation) improves the bioavailability, safety and prolongs circulation time in the blood of therapeutic agents [10]. Since NPs permeate through the leaky tumor vasculature to a higher degree than through the healthy tissue and remain in the area, they can be used for signaling or therapy [11] and, therefore, PEG is increasingly used to improve the properties of nanoparticles used as therapeutic or diagnostic agents in biomedicine.

In addition, nanoparticles suspended in other liquids had the tendency to agglomeration. Nanoparticles aggregate when the attraction between particles is stronger than the attraction with solvent particles. PEG decreases the surface energy of NPs and minimizes van der Waals attraction [10]. In addition, PEG is a very effective passivation agent and surface functionalization (passivation) of carbon nanoparticles is necessary to make them fluorescent. Although the origin of fluorescence from carbon nanoparticles is still not completely clear the fluorescence properties of carbon dots are associated with the particles' surface, specifically "surface defects" responsible for light absorbance. The photoluminescence originates from the surface functional 
groups attached to carbon nanodots and functionalization with hydrocarbon chains such as in PEG is very effective [5].

However, the experiments with laser synthesis in PEG are rather scarce $[3,5,12,13]$ and most of them were made in graphite powder [3, 12, 13]. For carbon dots produced by laser ablation of a carbon target in the presence of water vapor photoluminescence was achieved only after dots' surface passivation [5]. In this paper, the results of the synthesis of the fluorescent carbon dots (CDots) from solid carbon target and reduced graphene oxide ( $\mathrm{rGO}$ ) nanoparticles performed by the laser ablation in $\mathrm{PEG}_{200}$ are presented.

\section{Synthesis}

Graphite target irradiation was performed in a quartz beaker with diameter $40 \mathrm{~mm}$ using an Nd:YAG laser (Quantel, 981 E). The laser operated at a wavelength of 1064 and $355 \mathrm{~nm}$ with a $10 \mathrm{~ns}$ pulse duration and repetition rate of $10 \mathrm{~Hz}$. The thickness of liquid layer over the target was about $4 \mathrm{~mm}$. In the case of $355 \mathrm{~nm}$ radiation the laser fluence was 3.5-15 $\mathrm{J} \mathrm{cm}^{-2}$. In the first step of synthesis the target was irradiated for $15 \mathrm{~min}$. In the case of $1064 \mathrm{~nm}$, the maximum fluence during the ablation was $3.5 \mathrm{~J} \mathrm{~cm}^{-2}$ because greater fluence resulted in a shock wave and splashes of the liquid. The color of resulting suspensions was very different-after $355 \mathrm{~nm}$ radiation the suspension was still transparent while after $1064 \mathrm{~nm}$ radiation its color became brownish, which provides that the production of CNPs was much higher. After initial $15 \mathrm{~min}$ of ablation the obtained suspension was transferred to another beaker (20 $\mathrm{mm}$ in diameter) and $15-\mathrm{mm}-$ high suspension column was further irradiated (second step), either at a fluence of $15 \mathrm{~J} \mathrm{~cm}^{-2}$ in the case of $355 \mathrm{~nm}$ or at a fluence of $7 \mathrm{~J} \mathrm{~cm}^{-2}$ in the case of $1064 \mathrm{~nm}$ (in this case higher fluence again resulted in splashes of the liquid). All irradiations were made in an ultrasonic bath.

After the second step suspension irradiated with $355 \mathrm{~nm}$ radiation was still transparent. In the case of $1064 \mathrm{~nm}$ the color of the suspension became yellowish. It is worth mentioning that we performed the synthesis of nanoparticles by the laser ablation in various liquids such as deionized water, isopropyl alcohol (IPA), acetone and $\mathrm{PEG}_{200}$. The carbon nanoparticles synthesized in all these liquids, with exception of water, exhibited considerable fluorescent emission.

Similar procedure was applied in the case of rGO. Reduced graphene oxide was prepared using the method described previously [14]. Obtained graphene flakes were filtered and washed with deionized water to remove the by-products of the synthesis till the $\mathrm{pH}$ of the filtrate reached 6.5. Reduced graphene oxide rGO $(0.1 \mathrm{~g}$ of dry powder) was mixed with $40 \mathrm{ml}$ of $\mathrm{PEG}_{200}$ and milled in a planetary ball mill for $18 \mathrm{~h}$. Next the suspension was centrifuged for $30 \mathrm{~min}$ at a speed of $18,000 \mathrm{rpm}$. Finally the centrifuged part of suspension was diluted 1:100 in $\mathrm{PEG}_{200}$ and was irradiated by the laser beam at a $1064 \mathrm{~nm}$ wavelength-initially for $15 \mathrm{~min}$ at a fluence of $3.5 \mathrm{~J} \mathrm{~cm}^{-2}$ and next at a fluence of $7 \mathrm{~J} \mathrm{~cm}^{-2}$.

\section{Results and discussion}

The analysis of synthesized nanoparticles was made using the absorption and fluorescence spectroscopy as well as transmission electron microscopy (TEM), high-resolution TEM (HRTEM), and dynamic light scattering (DLS) technique. The photoluminescence spectra were taken with the use of inverted confocal microscope Leica TCS SP5 X as well as with a fluorescent microscope Leica DMI3000B connected with an HR4000 Ocean Optics spectrometer.

The absorbance of carbon nanoparticles was measured with a spectrophotometer (Thermo Scientific Multiscan $\mathrm{GO})$. The absorbance of CNPs was obtained from the suspension by subtracting the absorbance of pure $\mathrm{PEG}_{200}$. It has been checked that the irradiation of the pure PEG does not change its absorbance. The absorbance $A$ of obtained CNPs after 15 min of ablation is shown in Fig. 1. Further irradiation of the suspension does not change the absorbance substantially.

After 15 min of ablation the peak of absorbance is at $230 \mathrm{~nm}$ but practically in the range $215-320 \mathrm{~nm}$ whole incident radiation is absorbed on $1 \mathrm{~cm}$ path (Multiscan GO cuvette size). Since such high absorption does not give relevant information about the absorbance shape in the case of $1064 \mathrm{~nm}$ wavelength, the sample was diluted 1:10 in $\mathrm{PEG}_{200}$ and absorbance was measured again. The shape of absorbance curve is similar for both cases, 355 and $1064 \mathrm{~nm}$; the

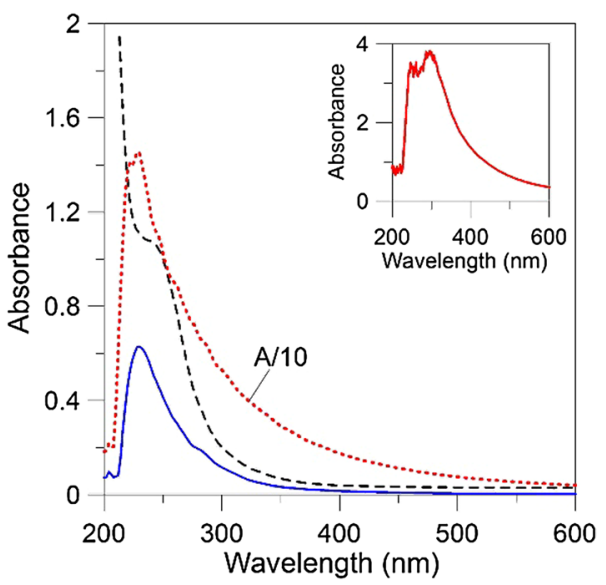

Fig. 1 Absorbance of suspension of CNPs in PEG $_{200}$ after 15 min of ablation; solid line-sample obtained with $355 \mathrm{~nm}$ laser pulse, dotted line-sample obtained with $1064 \mathrm{~nm}$ laser pulse (diluted), broken line-PEG. Inset shows absorbance of undiluted $1064 \mathrm{~nm}$ sample 
peak is around $230 \mathrm{~nm}$ but in the case of $1064 \mathrm{~nm}$ the distribution is broader.

The absorption of sample produced with $1064 \mathrm{~nm}$ wavelength is incomparably higher as a result of much greater concentration of nanoparticles. The Beer-Lambert law links the intensity attenuation of optical radiation through a sample to the density $N$ of species present in it.

$I_{T}(\lambda)=I_{0}(\lambda) e^{-N \sigma_{\lambda} d}=I_{0}(\lambda) e^{-\alpha_{\lambda} d}$

where $I_{T}(\lambda)$ is the intensity of transmitted light, $I_{0}$ is the intensity of light incident on the sample, $\sigma(\lambda)$ the absorption cross section, $d$ is the absorption length, and $\alpha(\lambda)$ is the absorption coefficient. The density of absorbing species in a sample can be determined from the value of the absorbance $A$,

$A=-\log \frac{I_{T}}{I_{0}}=N \sigma_{\lambda} d$.

Since the absorption cross section is proportional to square diameter of absorbing particles and DLS showed similar diameters of carbon nanoparticles produced with 355 and $1064 \mathrm{~nm}$ laser radiation, the much smaller absorbance in the case of $355 \mathrm{~nm}$ indicates smaller density of absorbing particles.

The very small output of nanoparticles with the $355 \mathrm{~nm}$ wavelength laser radiation at fluences of $3.5-4 \mathrm{~J} \mathrm{~cm}^{-2}$ is surprising. These results are essentially different from those obtained in vacuum when the ablation rate at 355 and $1064 \mathrm{~nm}$ was similar at fluences below $10 \mathrm{~J} \mathrm{~cm}^{-2}[15]$.

The measured absorbance of $\mathrm{PEG}_{200}$ was used to calculate the absorption of the laser beam in a liquid layer over the target. The absorption coefficient was calculated from the formula $\alpha=2.3026 \cdot A / d$ where $A$ is the absorbance and $d$ the sample thickness ( $1 \mathrm{~cm}$ in our case). The calculation using Lambert-Beer law showed that at $355 \mathrm{~nm}$ the laser beam is attenuated merely by $6 \%$ during ablation in a $4-\mathrm{mm}$ PEG layer. Such result does not explain such small ablation from the target in comparison with $1064 \mathrm{~nm}$ wavelength and suggests that ablation rates in liquids are considerably different from those in vacuum, especially in such a dense and viscous liquid such as PEG.

However, at large fluences $\mathrm{F}>10 \mathrm{~J} \mathrm{~cm}^{-2}$ the ablation mechanism in the case $355 \mathrm{~nm}$ changes to explosive boiling [15] or fragmentation [16]. Contrary to thermal evaporation such mechanisms probably do not lead to the nanoparticle formation. Indeed, after ablation with $355 \mathrm{~nm}$ at a fluence of $15 \mathrm{~J} \mathrm{~cm}^{-2}$ several carbon micro-fragments on the beaker bottom were observed, which explains a low output of nanoparticles.

Further analysis concerns only results were obtained at $1064 \mathrm{~nm}$ wavelength.

The photoluminescence spectra were taken with the use of an inverted confocal microscope Leica TCS SP5 X as well as with a fluorescent microscope Leica DMI3000B connected with an HR4000 Ocean Optics spectrometer. The excitation wavelengths were $405,488,561,633 \mathrm{~nm}$ and $370,405,470,550 \mathrm{~nm}$, respectively. All suspensions resulting from $1064 \mathrm{~nm}$ ablation exhibited a typical strong broadband photoluminescence with a maximum dependent on the excitation wavelength. Although its origin is not yet fully understood, there is rising evidence that it is extrinsic fluorescence resulting from surface states. Indeed, the close relationship between functional groups of ligands attached to the CDots surface and carbon dots luminescence has been demonstrated in many studies [5, 17-19].

The results for the sample obtained after 15 min ablation with $1064 \mathrm{~nm}$ laser pulse and additional $1 \mathrm{~h}$ irradiation of the suspension are presented in Fig. 2. It is worth noting that photoluminescence spectra were similar in the case of $355 \mathrm{~nm}$ radiation although the emission was much smaller due to low concentration of nanoparticles. On the other hand, contrary to expectations, when the first step was made using $1064 \mathrm{~nm}$ wavelength the change of the wavelength in the second step to $355 \mathrm{~nm}$ wavelength did not improve the results. This was due to much higher absorption coefficient of $355 \mathrm{~nm}$ which resulted in a poor penetration of the 15-mm-high suspension. Calculation shows that the incident $355 \mathrm{~nm}$ radiation is attenuated by a factor of 5 on the path of $5 \mathrm{~mm}$.

Figure 3 shows the FTIR spectra of suspension of CNPs suspended in $\mathrm{PEG}_{200}$. The whole spectrum together with the position of vibrational absorption band of carboxylate group $\mathrm{C}=\mathrm{O}$ at $1647 \mathrm{~cm}^{-1}$ is very similar to that presented by Castro et al. [12]. The intrinsic features of CDots spectrum are overshadowed by the absorption of PEG and can be well

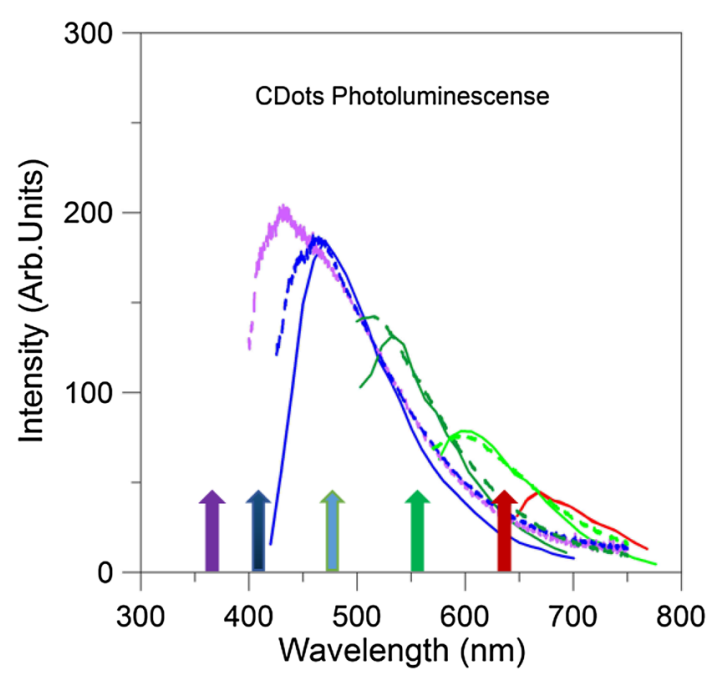

Fig. 2 Photoexcitation and photoluminescence spectra of carbon nanoparticles (CNPs) suspended in $\mathrm{PEG}_{200}$. Excitation wavelengths are indicated by arrows; solid line-inverted confocal microscope, broken line-fluorescent microscope 


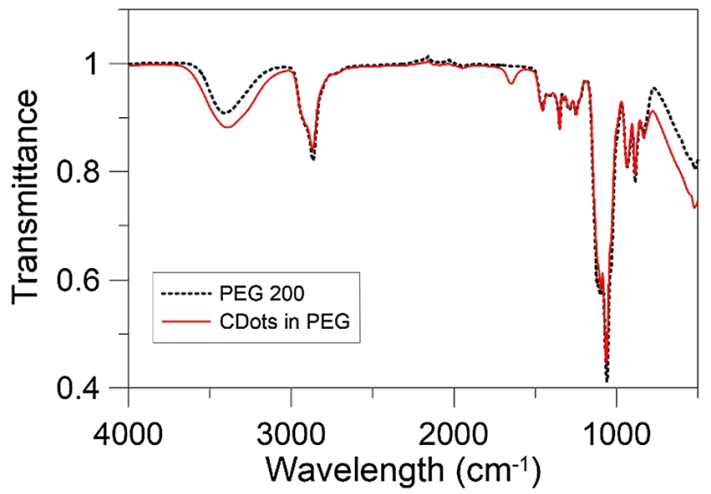

Fig. 3 FTIR spectra; solid line-suspension of CDots in $\mathrm{PEG}_{200}$, dotted line $-\mathrm{PEG}_{200}$

seen only where the IR absorption of PEG is close to zero. The intensity of the $\mathrm{C}=\mathrm{O}$ band clearly increases with the concentration of CDots.

The size of carbon particles was measured by dynamic light scattering technique (DLS) using Zetasizer Nano ZS
(Malvern Instruments Ltd., UK). The Zetasizer system measures Brownian motion and relates it to the particle size. Samples were measured in different media: acetone, water, and PEG 200. The measuring chamber of Zetasizer was kept at constant temperature of $25{ }^{\circ} \mathrm{C}$ for samples prepared in acetone and water, and $20^{\circ} \mathrm{C}$ for samples prepared in PEG 200. Measurements were performed five times for each sample with automatic detection of number of runs, and results are presented as average size distribution by number of particles in the sample (histograms). The results are shown in Fig. 4 . The additional $1 \mathrm{~h}$ irradiation significantly shifted the distribution towards few nanometer size particles. This result is supported by the HRTEM images shown in Fig. 5. The observations were taken with Scanning Transmission Electron Microscope HITACHI HD2700 (STEM) at accelerating voltage for $200 \mathrm{keV}$. Sample was prepared by deposition of carbon particles on the surface of thin carbon film of TEM grid. To avoid contamination in the microscope the sample was treated in plasma cleaner with $\mathrm{H}-\mathrm{O}$ gas mixture for $20 \mathrm{~s}$. The images were made in a bright field (BF) and in a high angle annular dark field (HAADF). The image 5a was taken
Fig. 4 Particle size distribution from the dynamic light scattering (DLS) method: a after $15 \mathrm{~min}$ of ablation $(1064 \mathrm{~nm}$, $F=3.5 \mathrm{~J} \mathrm{~cm}^{-2}$ ), b after $1 \mathrm{~h}$ of additional irradiation of the suspension with $1064 \mathrm{~nm}$ radiation at $F=7 \mathrm{~J} \mathrm{~cm}^{-2}$
Fig. 5 HRTEM images after additional irradiation; a BFTEM, b HAADF-STEM
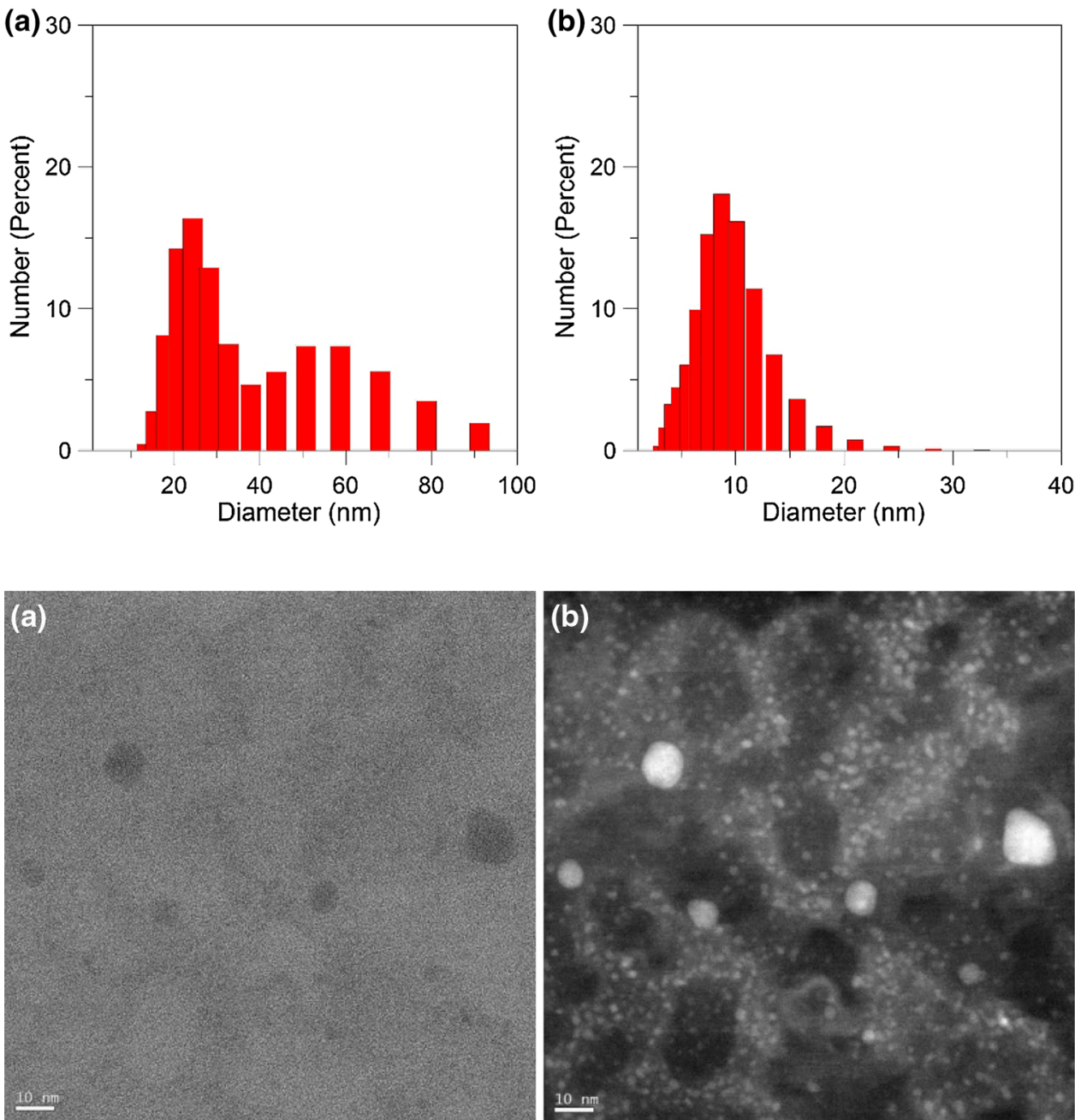
in a bright field where the atomic mass contrast is weak, and only thicker carbon particles are well visible. The image $5 \mathrm{~b}$ was taken with HAADF detector and represents density contrast. Scattered electrons were collected with annular detector; therefore, even small changes in density are well visible. This picture shows prevailing presence of carbon dots. The particle size distribution obtained from HRTEM images is presented in Fig. 6. The results acquired from HRTEM images show somewhat smaller size nanoparticle size than the DLS measurements.

Similar results were obtained in the case of laser-modified rGO. Figure 7 shows the absorbance of rGO nanoparticles. The absorbance of rGO changed dramatically after laser irradiation. Second step irradiation did not change the qualitative character of the absorbance. Quantitatively the shape is different because of greater concentration of absorbing particles.

The photoluminescence spectra after laser irradiation (Fig. 8) are similar to those obtained in the case of carbon dots but are several times weaker. However, as indicated in Fig. 9 very small nanoparticles were not obtained after the two-step laser irradiation, although the particle size distributions have been considerably shifted towards smaller diameters. In fact, HRTEM images (Fig. 10) showed even smaller particles-with diameter about $10 \mathrm{~nm}$.

\section{Conclusions}

Two-step irradiation applied in the case of graphite target resulted in a very effective production of carbon nanodots. However, the ablation in PEG appeared to be effective only in the case of $1064 \mathrm{~nm}$ laser pulse, the ablation with $355 \mathrm{~nm}$ resulted in a surprisingly small production rate of nanoparticles. The very small output of nanoparticles with a $355 \mathrm{~nm}$ laser radiation at fluences $3.5-4 \mathrm{~J} \mathrm{~cm}^{-2}$ shows that ablation

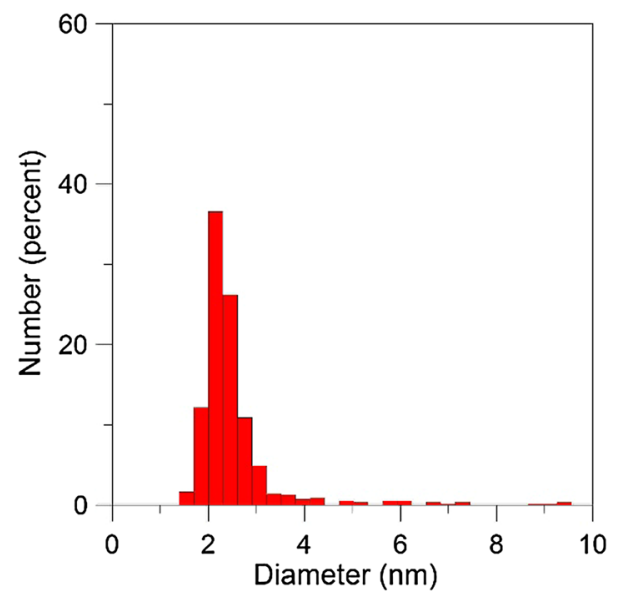

Fig. 6 Particle size distribution derived from the HRTEM images

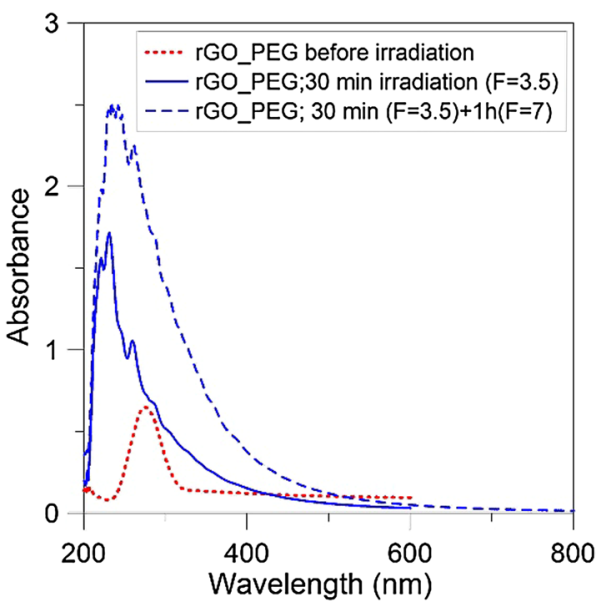

Fig. 7 Absorbance of suspension of $\mathrm{rGO}$ in $\mathrm{PEG}_{200}$; dotted linebefore irradiation, solid line-after $15 \mathrm{~min}$ irradiation at $F=3.5$ $\mathrm{J} \mathrm{cm} \mathrm{cm}^{-2}$, broken line-after $1 \mathrm{~h}$ of additional irradiation at $F=7$ $\mathrm{J} \mathrm{cm}^{-2}$

rate in liquid, specifically in such a dense and viscous liquid like PEG, is considerably different from that in vacuum. At large fluences $F \geq 10 \mathrm{~J} \mathrm{~cm}^{-2}$ the ablation mechanism is apparently explosive boiling and does not lead to nanoparticle formation. In the second step the irradiation with a $355 \mathrm{~nm}$ wavelength was not effective due to absorption of the laser radiation on a short path.

The same two-step irradiation procedure was less effective in the case of rGO and resulted in larger particles.

In both cases, produced nanoparticles exhibited strong, broadband photoluminescence with a maximum dependent on the excitation wavelength. The diameters of nanoparticles and their distributions were determined from the DLS

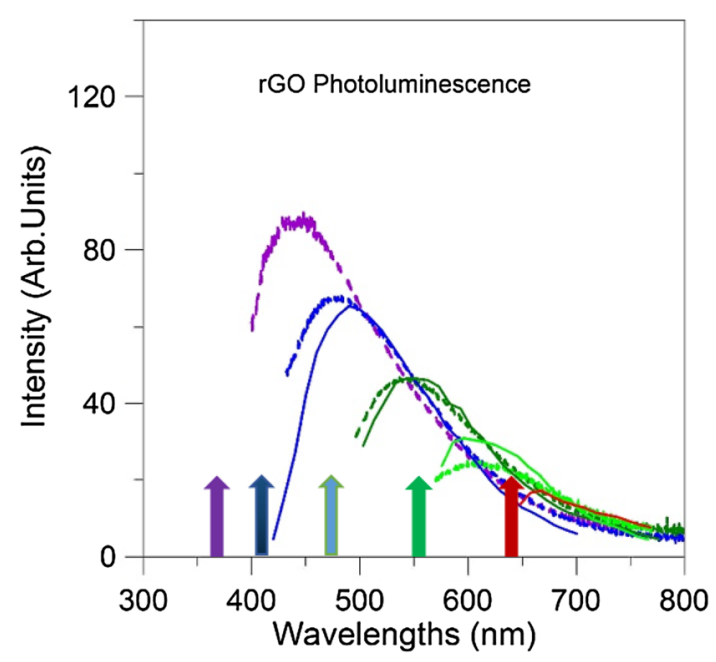

Fig. 8 Photoexcitation and photoluminescence spectra of $\mathrm{rGO} /$ $\mathrm{PEG}_{200}$ suspension after second step of laser irradiation. Excitation wavelengths are indicated by arrows 

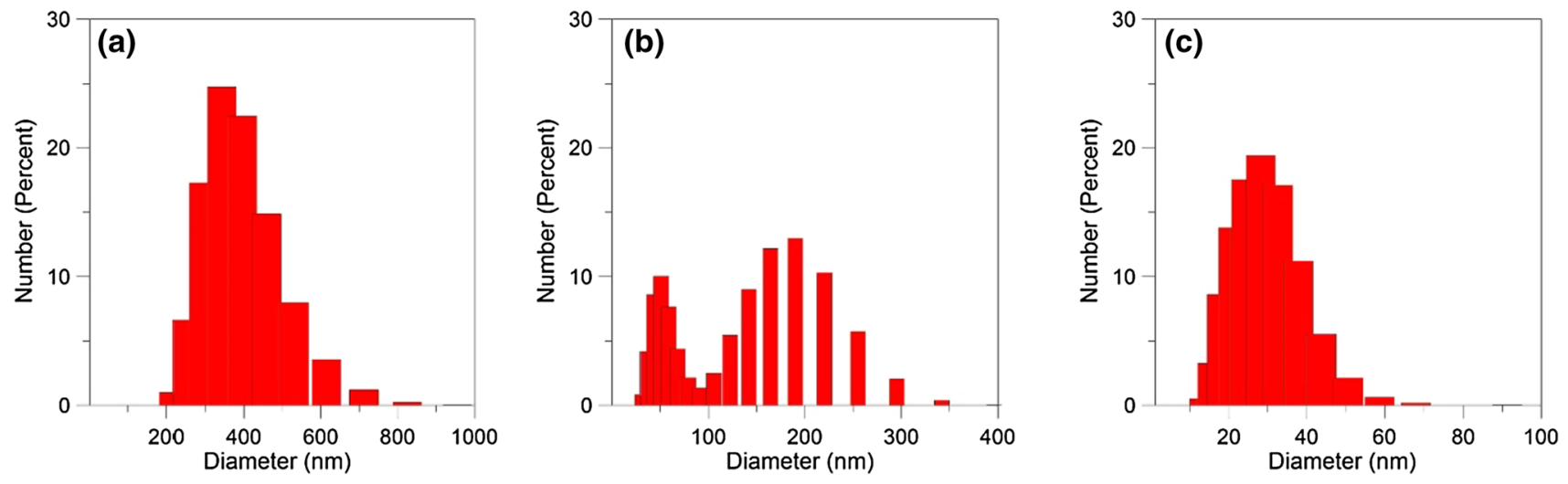

Fig. 9 Particle size distribution by dynamic light scattering (DLS) method: a before laser irradiation, $\mathbf{b}$ after 15 min irradiation at $F=3.5 \mathrm{~J} \mathrm{~cm}{ }^{-2}$, c after $1 \mathrm{~h}$ of additional irradiation at $F=7 \mathrm{~J} \mathrm{~cm}^{-2}$

Fig. 10 a HRTEM images of rGO nanoparticles after additional irradiation, b corresponding particle size distribution
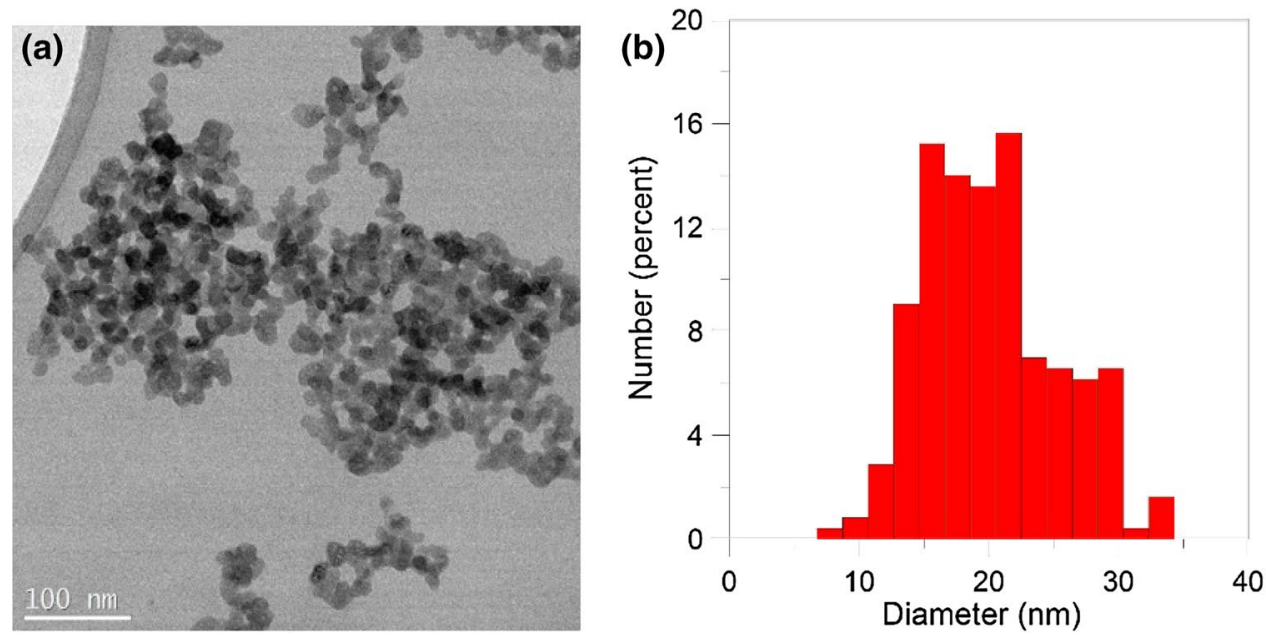

measurements and HRTEM images. The agreement between both methods was fair although the DLS provided higher particle size than the HRTEM measurements.

Acknowledgements This work was supported by the Polish National Science Centre (Research Project 2014/13/B/ST8/04290).

Open Access This article is distributed under the terms of the Creative Commons Attribution 4.0 International License (http://creativeco mmons.org/licenses/by/4.0/), which permits unrestricted use, distribution, and reproduction in any medium, provided you give appropriate credit to the original author(s) and the source, provide a link to the Creative Commons license, and indicate if changes were made.

\section{References}

1. N.G. Semaltianos, Crit. Rev. Solid State Mater. Sci. 35, 105-124 (2010)
2. D. Zhang, B. Gökce, S. Barcikowski, Chem. Rev. 117, 3990-4103 (2017)

3. S.-L. Hu, K.-Y. Niu, J. Sun, J. Yang, N.-Q. Zhao, X.-W. Du, J. Mater. Chem. 19, 484-488 (2009)

4. S.K. Shin, J.K. Song, S.M. Park, Appl. Surf. Sci. 257, 5156-5158 (2011)

5. Y.-P. Sun, B. Zhou, Y. Lin, W. Wang, K.A.S. Fernando, P. Pathak, M. J.Meziani, B.A. Harruff, X. Wang, H. Wang, P.G. Luo, H. Yang, M.E. Kose, B. Chen, L.M. Veca, S.-Y. Xie, J. Am. Chem. Soc. 128(24), 7756-7757 (2006)

6. O.S. Wolfbeis, Chem. Soc. Rev. 44, 4743-4768 (2015)

7. V. Nguyen, J. Si, L. Yan, X. Hou, Carbon 108, 268-273 (2016)

8. D. Reyes, M. Camacho, M. Camacho, M. Mayorga, D. Weathers, G. Salamo, Z. Wang, A. Neogi, Nanoscale Res. Lett. 11, 424 (2016)

9. H.P.S. Castro, V.S. Souza, J.D. Scholten, J.H. Dias, J.A. Fernandes, F.S. Rodembusch, R. dos Reis, J. Dupont, S.R. Teixeira, R.R.B. Correia, Chem. Eur. J. 22, 138-143 (2016)

10. J.V. Jokerst, T. Lobovkina, R.N. Zare, S.S. Gambhir, Nanomedicine (Lond). 6(4), 715-728 (2011)

11. K. Shameli, M. Bin Ahmad, S.D. Jazayeri, S. Sedaghat, P. Shabanzadeh, H. Jahangirian, M. Mahdavi, Y. Abdollahi, Int. J. Mol. Sci. 13, 6639-6650 (2012) 
12. H.P.S. Castro, M.K. Pereira, V.C. Ferreira, J.M. Hickmann, R.R.B. Correia, Opt. Mat. Express 7(2), 401-408 (2017)

13. V. Nguyen, L. Yan, J. Si, X. Hou, J. Appl. Phys. 117, 084304 (2015)

14. L. Stobinski, B. Lesiak, A. Malolepszy, M. Mazurkiewicz, B. Mierzwa, J. Zemek, P. Jiricek, I. Bieloshapka, J. Electron Spectrosc. Relat. Phenom. 195, 145-154 (2014)

15. J. Hoffman, J. Chrzanowska, S. Kucharski, T. Moscicki, I.N. Mihailescu, C. Ristoscu, Z. Szymanski, Appl. Phys. A 117, 395400 (2014)
16. J. Hoffman, J. Phys. D; Appl. Phys. 48, 235201 (2015)

17. S. Zhu, Y. Song, X. Zhao, J. Shao, J. Zhang, B. Yang, Nano Res. 8, 355-381 (2015)

18. L. Pan, S. Sun, A. Zhang, K. Jiang, L. Zhang, C. Dong, Q. Huang, A. Wu, H. Lin, Adv. Mater. 27, 7782-7787 (2015)

19. M. Fu, F. Ehrat, Y. Wang, K.Z. Milowska, C. Reckmeier, A.L. Rogach, J.K. Stolarczyk, A.S. Urban, J. Feldmann, Nano Lett. 15 6030-6035 (2015) 